\title{
NONUNIQUENESS OF BEST APPROXIMATING COMPLEX RATIONAL FUNCTIONS
}

\author{
BY E. B. SAFF ${ }^{1}$ AND R. S. VARGA ${ }^{2}$
}

Communicated by Alston S. Householder, November 2, 1976

1. Introduction. For any real continuous function $f(x)$ on $[-1,1]$ and for any nonnegative integer $n$, it is well known that the best uniform approximation to $f(x)$ on $[-1,1]$ by a real rational function of order $n$ is unique (cf. [1]). On the other hand, J. L. Walsh [3] has given an example of a continuous complex-valued function on a certain compact crescent-shaped region in the plane, whose best uniform complex rational approximation of order 1 is not unique. In this note we present results which show that this nonuniqueness of best complex rational approximations can hold even in the case of approximating real functions on finite real intervals. The examples given below point out a somewhat unexpected fact; namely, that complex rational functions may yield closer uniform approximations to a real function on $[-1,1]$ than the best real rational function of the same type. This is in marked contrast with the theory for best complex polynomial approximation.

In $\S 2$ we will state the main results and examples. The proofs, further details, and related open problems will be published elsewhere [2]. For the remainder of this section we introduce the necessary notation.

For any integer $n \geqslant 0$, we let $\pi_{n, n}^{r}$ denote the collection of all rational functions which can be written in the form $p / q$, with $p$ and $q$ polynomials of degree at most $n$ having real coefficients. We use $\pi_{n, n}^{c}$ to denote the analogous collection of such rational functions with $p$ and $q$ having complex coefficients.

If $C_{r}[-1,1]$ denotes the collection of all real continuous functions on $[-1,1]$, and if $\|g\|:=\sup \{|g(x)| ;-1 \leqslant x \leqslant 1\}$ for any real- or complex-valued function $g$ defined on $[-1,1]$, then we further set

$$
E_{n, n}^{r}(f):=\inf _{g \in \pi_{n, n}^{r}}\|f-g\| ; \quad E_{n, n}^{c}(f):=\inf _{g \in \pi_{n, n}^{c}}\|f-g\|,
$$

for any $f \in C_{r}[-1,1]$. Obviously, $E_{n, n}^{c}(f) \leqslant E_{n, n}^{r}(f)$. The collection of all

AMS (MOS) subject classifications (1970). Primary 41A20; Secondary 41A50.

${ }^{1}$ Research supported in part by the Air Force Office of Scientific Research under Grant AFOSR-74-2688.

${ }^{2}$ Research supported in part by the Air Force Office of Scientific Research under Grant AFOSR-74-2729, and by the Energy Research and Development Administration (ERDA) under Grant E(11-1)-2075. 
best approximating rational functions from $\pi_{n, n}^{r}$ (or $\pi_{n, n}^{c}$ ) to a given $f \in C_{r}[-1,1]$ is denoted by $B_{n, n}^{r}(f)\left(\right.$ or $\left.B_{n, n}^{c}(f)\right)$, i.e.,

$$
\begin{aligned}
& B_{n, n}^{r}(f):=\left\{r \in \pi_{n, n}^{r}:\|f-r\|=E_{n, n}^{r}(f)\right\} \\
& B_{n, n}^{c}(f):=\left\{r \in \pi_{n, n}^{c}:\|f-r\|=E_{n, n}^{c}(f)\right\},
\end{aligned}
$$

and the cardinality of these sets is symbolized by $\left|B_{n, n}^{r}(f)\right|\left(\right.$ or $\left.\left|B_{n, n}^{c}(f)\right|\right)$. As previously remarked, $\left|B_{n, n}^{r}(f)\right|=1$, and its unique element is denoted by $R_{n, n}(f)=R_{n, n}$. It is known [3] that $B_{n, n}^{c}(f) \neq \varnothing$. Our concern is with conditions which ensure that $\left|B_{n, n}^{c}(f)\right|>1$, that is, when nonuniqueness holds for best uniform rational approximations in $\pi_{n, n}^{c}$ to a real $f$ on $[-1,1]$.

2. Some results and examples.

Proposition 1. Given $f \in C_{r}[-1,+1]$, let $R_{n, n}=P / Q \in B_{n, n}^{r}(f)$, and set $d:=\max (\operatorname{deg} P ; \operatorname{deg} Q)$, where $P$ and $Q$ have no common factors. If $f-R_{n, n}$ has an alternation set on $[-1,1]$ of length at least $2+2 n+d$, then $B_{n, n}^{c}(f)=B_{n, n}^{r}(f)=\left\{R_{n, n}\right\}$.

Proposition 2. Given $f \in C_{r}[-1,+1]$, let $R_{n, n}=P / Q \in B_{n, n}^{r}(f)$ and let $d:=\max (\operatorname{deg} P ; \operatorname{deg} Q)$ where $P$ and $Q$ have no common factors. If every alternation set on $[-1,1]$ for $f-R_{n, n}$ has length at most $1+2 n-d$, then

$$
E_{n, n}^{c}(f)<E_{n, n}^{r}(f) \text {, and }\left|B_{n, n}^{c}(f)\right|>1 \text {. }
$$

The above propositions are used to justify the following examples.

ExAmple 1. Let $f \in C_{r}[-1,+1]$ be even, and monotone and nonconstant on $[0,1]$. Then,

$$
E_{1,1}^{c}(f)<E_{1,1}^{r}(f), \text { and }\left|B_{1,1}^{c}(f)\right|>1
$$

We remark that Example 1 includes as a special case the function $f(x):=$ $|x|^{\alpha}, \alpha>0$, on $[-1,+1]$.

ExAmPle 2. For $n>1$, let $T_{2 n-1}(x)$ be the Chebyshev polynomial (of the first kind) of degree $2 n-1$. Then, for each $k$ with $0 \leqslant k \leqslant n-1$, $\left|B_{k, k}^{c}\left(T_{2 n-1}\right)\right|=1$, while for $k=n$,

$$
E_{n, n}^{c}\left(T_{2 n-1}\right)<E_{n, n}^{r}\left(T_{2 n-1}\right), \text { and }\left|B_{n, n}^{c}\left(T_{2 n-1}\right)\right|>1
$$

Example 3. There exists an entire function $f \in C_{r}[-1,+1]$ and an infinite sequence of distinct positive integers $\left\{n_{i}\right\}_{i=1}^{\infty}$ such that

$$
E_{n_{i}, n_{i}}^{c}(f)<E_{n_{i}, n_{i}}^{r}(f), \text { and }\left|B_{n_{i}, n_{i}}^{c}(f)\right|>1, \quad \forall i \geqslant 1 \text {. }
$$




\section{REFERENCES}

1. G. Meinardus, Approximation of functions: Theory and numerical methods, Springer-Verlag, New York, 1967. MR 36 \#571.

2. E. B. Saff and R. S. Varga, Nonuniqueness of best complex rational approximations to real functions on real intervals, J. Approximation Theory (to appear).

3. J. L. Walsh, Interpolation and approximation by rational functions in the complex domain, 5th ed., Amer. Math. Soc. Colloq. Publ., Vol. 20, Amer. Math. Soc., Providence, R. 1., 1966.

DEPARTMENT OF MATHEMATICS, UNIVERSITY OF SOUTH FLORIDA, TAMPA FLORIDA 33620

DEPARTMENT OF MATHEMATICS, KENT STATE UNIVERSITY, KENT, OHIO 44242 